Acta Hispanica (2020) Supplementum II: 847-858

\title{
AFIRMACIÓN DE SUBJETIVIDADES DE LOS PROFESORES DE INGLÉS EN COLOMBIA A PARTIR DE SUS DISCURSOS DE RESISTENCIA $^{1}$
}

\section{Eliana GaRzón DUARTE}

\author{
Universidad de Szeged - Universidad Distrital Francisco José de Caldas
}

\begin{abstract}
Resumen: Este artículo tiene como objetivo presentar los resultados de una investigación llevada a cabo por un grupo de profesores de la Universidad Distrital Francisco José de Caldas, en Bogotá, Colombia. La investigación se centró en problematizar el significado de subjetividad construido por los profesores de inglés en el territorio colombiano, como respuesta al Plan Nacional de Bilingüismo - proyecto diseñado e implementado por el Ministerio de Educación Nacional desde el año 2004, en Colombia. El doble carácter de la sujeción/subjetivación, planteado por Foucault (1982), es utilizado en esta investigación para analizar el trabajo de los profesores de inglés en cuanto a las prácticas del sí y las de resistencia o contestatarias, no solo para afirmarse ellos mismos como investigadores y pensadores críticos, sino también para reclamar reconocimiento como educadores que producen un saber relevante y situado. El Análisis Histórico de Discurso, a través de procedimientos arqueológicos (Foucault, 1972), se utilizó para rastrear las prácticas discursivas y no-discursivas de los profesores de inglés. Esta metodología fue clave para develar la manera cómo los profesores de inglés se identifican a sí mismos, su oposición a las políticas públicas de lenguaje y cómo responden ellos a los discursos dominantes en relación a la enseñanza del inglés. Más de 100 publicaciones académicas de profesores de inglés fueron revisadas y confrontadas con discursos normalizados que circulan en los programas de gobierno, medios de prensa y documentos de expertos. Los resultados de esta investigación contribuyen al entendimiento de los profesores de inglés en relación con sus propias luchas y el papel de sus prácticas de resistencia para afirmar sus subjetividades.
\end{abstract}

Palabras clave: subjetividad, resistencia, bilingüismo, políticas, prácticas discursivas.

Abstract: This paper aims to present the results of a research study carried out by a group of teachers at Universidad Distrital Francisco José de Caldas, in Bogotá, Colombia. The study focused on problematizing the meaning of subjectivity constructed by Colombian English Teachers in response to a National Bilingual Program — plan designed and implemented by the Ministry of Education since 2004. The double-side character of subjection/subjectification (Foucault, 1982) is used to analyze English teachers' work of the self and contestarian/resistance practices to affirm themselves as researchers and critical thinkers but also to claim recognition as educators who produce relevant and situated knowledge. Historical Discourse Analysis, through archeological procedures (Foucault, 1972) to trace back English teachers' discursive and nondiscursive practices, were key to unveiling how teachers think of themselves as English teachers,

1 Agradecimientos. Esta investigación fue posible gracias al grupo de investigadores y coinvestigadores que trabajaron en el proyecto: Dra. Pilar Méndez Rivera, Mg. Rodolfo NoriegaBorja, estudiantes Francisco Guillermo Rodríguez Motavita y Germán Alexis Osorio Junca.

Acta Hispanica, Hungría, Supplementum II: 847-858, 2020, ISSN: 1416-7263 | 847 


\section{Afirmación de subjetividades de los profesores de inglés en Colombia \\ a partir de sus discursos de resistencia}

oppose policies and respond to dominant discourses in relation to English teaching. More than 100 English teachers' academic publications were revised and confronted with normalized discourses circulating in political programs, print media and experts' documents. Findings contribute to EFL teachers' understanding of their own struggles and the role of their resistance practices to affirm their subjectivities.

Keywords: Subjectivity, Resistance, Bilingualism, Policies, Discursive Practices.

\section{Introducción}

El Plan Nacional de Bilingüismo en Colombia (PNB) ha sido un proyecto implementado por el Ministerio de Educación Nacional desde el año 2004. Este proyecto tiene como objetivo principal formar ciudadanos capaces de comunicarse en inglés, utilizando los estándares internacionales del Marco Común Europeo de Referencia para las Lenguas para integrar al país en los procesos de globalización y apertura cultural. El PNB busca que, a partir de 2019, los estudiantes terminen su educación media con un nivel B1 y que los profesores de inglés alcancen un nivel, por lo menos, de B2. Desde el inicio de implementación del PNB, han sido numerosos los debates que han surgido en torno a la forma como se ha desarrollado este proyecto dentro del contexto particular del país. Los resultados obtenidos con la implementación de este Plan han sido presentados a través de reportes estadísticos que contrastan el progreso en marcha con la inversión de presupuesto y las prácticas discursivas entrelazadas con ideas de productividad y competitividad como medidas de eficacia social. En estos reportes estadísticos, la figura del profesor de inglés ha sido reducida a su nivel de lengua según los lineamientos del Marco Común Europeo (A1, A2, B1, B2, C1, C2) o al número de profesores que han sido beneficiados por programas de entrenamiento en el fortalecimiento de lengua, pero poco se ha dicho sobre los esfuerzos que los profesores de inglés deben hacer mientras se lleva a cabo la implementación del PNB en sus aulas y las innumerables dificultades que deben enfrentar en su quehacer pedagógico para lograr los objetivos allí planteados. Algunos de los efectos del PNB han sido las percepciones erróneas que de los profesores de inglés ha construido la sociedad del país. Una de las causas de esta negligencia se deriva de las prácticas de normalización que favorecen el dominio del lenguaje, cosificando una identidad nativa del inglés y haciendo invisible otros aspectos constituyentes de ser un profesor en nuestro país, lo cual fue repetido como dogma en la retórica de una reforma educativa y política, afectando las maneras en que los profesores de inglés son percibidos como sujetos deficitarios. Así pues, uno de los aspectos centrales de este estudio es la preocupación por la construcción de las subjetividades de los profesores de inglés en relación con la consideración dominante idealizada de cómo un profesor de inglés debe ser a través de lo que se plantea en las políticas y discursos dominantes. 
En este sentido, esta investigación considera la relación entre subjetividad y políticas a través de varios lentes: muestra las formas en que las concepciones de las políticas lingüísticas en relación con el bilingüismo sirvieron para producir un tipo de profesor de inglés meramente técnico que cumple con las prácticas de mercado; trae a la superficie las fuerzas confrontantes de poder y conocimiento entre los discursos de dominación y resistencia en la enseñanza del inglés; y busca comprender las dificultades de los profesores de inglés para refutar y desmantelar un sistema de razonamiento que los minimiza y trata de robar sus condiciones de posibilidad de pensar, actuar y ser diferentes. Para esto, la investigación se realizó en torno a las siguientes preguntas: ¿Cómo se han constituido los profesores de inglés como sujetos? ¿Cómo ha influido el PNB en la construcción de imagen de los profesores de inglés? ¿Qué piensan los profesores de inglés sobre ellos mismos?

Para dar respuesta a estas preguntas de investigación, se hizo una revisión de la relación entre política y subjetividad a través de los efectos del Plan Nacional de Bilingüismo, al igual que una revisión arqueológica que ayudó a rastrear un evento importante en la historia de la enseñanza de las lenguas en Colombia, el cual marcó el paso de concebir el aprendizaje del inglés como recurso cultural a convertirse en una estrategia de mercado de ciudadanía. Situación que también se puede representar en otros contextos internacionales donde el inglés es aprendido como lengua extranjera; por ejemplo, México, Chile y otros países del Tercer Mundo y colonizados. Este estudio trata de mostrar las luchas de los profesores de inglés para resistir este dominio de mercado y gobernanza económica (Lazzarato, 2014) en relación con las lenguas y la construcción de subjetividad para entender las prácticas discursivas y no discursivas de los profesores de inglés para pensar en sí mismos.

\section{Revisión de la literatura}

El estudio de la subjetividad ha sido dominado por teorías deterministas mientras los discursos dominantes operan como discursos de "deber ser" para asignarnos una identidad, un sexo, una profesión y una misión. Aunque los estudios de este tipo (por ejemplo, tecnologías de capitalismo, ver Guattari, 1996; economía subjetiva en Lazzarato, 2014; pensamiento occidental, ver Foucault, 2011) han sido importantes para comprender cómo la producción y construcción de subjetividades han servido a un sistema de razonamiento que normaliza el estudio de la subjetividad desde la perspectiva de la resistencia, esta ha sido relegada a un segundo plano para referirse a la gobernanza de su propia existencia en el campo de la enseñanza del inglés. La subjetividad es vista como una realidad empírica y una categoría analítica (Biehl, Good y Kleinman, 2007) no solo en el sentido de que puede ser trazada como una formación discursiva en los procesos históricos y contingentes de producción de identidades, sino también que puede ser experimentada como un proceso interior en el cual los sujetos luchan por interrumpir los modos impuestos de subjetivación para asignarse a ellos mismos una subjetividad 


\section{Afirmación de subjetividades de los profesores de inglés en Colombia \\ a partir de sus discursos de resistencia}

individual (Foucault, 1981). Al considerar las tecnologías del sí, Foucault (1988) explica cómo los individuos ejercen una serie de procedimientos para aceptar una identidad, mantenerla o transformarla, teniendo en cuenta cómo una nueva comprensión de un sujeto no esencial ilumina las formas en las que los individuos trabajan en ellos mismos para moldear sus subjetividades. Un eje importante de esta nueva comprensión tiene que ver con la heterogeneidad y contingencia de nuestras subjetividades a medida que se desarrollan dentro del ámbito de la experiencia (Kleinman y Fitz-Henry, 2007), el cual abarca no solo prácticas de poder-resistencia, sino también negociaciones, contradicciones y afirmaciones.

Es importante mencionar que el crecimiento del interés en la subjetividad desde las diferentes perspectivas (teorías feminista, psicoanalítica y posmoderna) ha impulsado en diferentes campos una clase de investigación que se centra en la actividad de auto reflexión de los individuos (Atkins, 2005) para entender cómo estos enfrentan las presiones y las dificultades del día a día, afectados por un sistema de razonamiento que promueve eficiencia, competencia e identidades normalizadas (técnicas de dominación) y tratan de entender cómo los individuos ejecutan algunas acciones (técnicas del sî) para alcanzar una existencia de autogobierno en la cual ellos encuentren sus propias aspiraciones. Aunque en el campo de la enseñanza del inglés el interés en identidad como categoría analítica ha servido para explorar algunos aspectos de la subjetividad (Castañeda-Peña y Ubaque, 2017; Viáfara, 2016; Guerrero y Quintero, 2016; Guerrero y Meadows, 2015; Pennington, 2015), pocos estudios han sido enfocados en analizarla como una práctica de auto entendimiento y autogobierno. En este sentido, esta investigación es una contribución al campo de la enseñanza del inglés desde la línea de constitución de sujetos, subjetividad y prácticas de resistencia para examinar las luchas de los profesores de inglés en Colombia en su búsqueda por dar sentido a su vida y trabajo en un contexto lleno de sospecha, crítica y escrutinio público.

En la revisión de los registros documentales, se pudo notar que hubo un giro en la manera como se entiende la enseñanza de lenguas extranjeras en Colombia. En el pasado, la enseñanza de lenguas tales como el latín, inglés, alemán y francés eran consideradas cursos especiales para la educación de estudiantes de secundaria, lo cual puede verificarse en el currículo de los períodos 1930-1950. Luego, desde 1950 hasta 1970, la enseñanza de lenguas solo se enfocó en inglés y francés, aunque latín podía ser estudiado como un curso opcional. Después de 1998, francés fue oficialmente removido del currículo y el reinado del inglés como lengua extranjera oficial en la educación empezó. Inglés fue introducido y continúa siendo promovido por los medios de comunicación como el lenguaje universal, el lenguaje del éxito profesional y el progreso social, el lenguaje de la tecnología y de las ciencias. Este giro es importante para problematizar la promoción de un sujeto bilingüe, que debe hablar inglés y español, con la noción de mercantilismo del sí (Lazzarato, 2014; Macedo et al., 2003), lo cual puede ser representado como un efecto del imperialismo para producir en los ciudadanos colombianos un deseo de trabajar sus subjetividades en relación directa al inglés. La revisión de estos programas educacionales 
y políticos introdujeron el inglés como una razón de mercado en un período particular de la historia de la sociedad colombiana, donde los profesores de inglés debían cooperar obedeciendo las políticas nacionales y las recomendaciones de expertos en competencia lingǘstica, promoviendo su identidad como profesores certificados que quieren ser evaluados con pruebas internacionales.

\section{Método de la investigación}

Este estudio se encuentra enmarcado en análisis histórico de discurso y basado en procedimientos arqueológicos para rastrear formaciones discursivas. Aunque hay diferentes maneras de conducir este tipo de investigación, este en particular tuvo en cuenta la perspectiva foucaultiana en la cual un gran número de registros fueron confrontados con discursos educativos, sociales y políticos para entender las maneras en las que algunas regularidades y discontinuidades surgen como parte de un dominio gubernamental. Los procedimientos arqueológicos basados en la lectura crítica y analítica son demandados como parte de una estrategia de develar la función enunciativa de una serie de signos, "una serie que no es necesariamente estructuras gramaticales o lógicas" (Foucault, 1972: 108), sino formaciones discursivas.

El análisis histórico presenta una organización relacional de declaraciones: "declaraciones diferentes en forma y dispersas en tiempo forman un grupo si ellas refieren a un mismo objeto único" (Foucault, 1972: 32). Al revisar la historia de la enseñanza de lenguas en Colombia, se trazaron registros de diferentes fuentes documentales, no solamente aquellas confinadas a la educación misma, sino también políticas, económicas, procesos sociales, patrones de comportamiento y sistemas de normas. La revisión de alrededor de 150 artículos de medios digitales y algunas versiones impresas de los medios, también la revisión de alrededor de 60 documentos oficiales del gobierno y del Consejo Británico, como expertos, y particularmente más de 120 números de revistas académicas para revisar los pronunciamientos de los profesores de inglés fueron leídos para reagrupar declaraciones en una amalgama de temas. Como parte de la articulación temática, el inglés como lengua de aprendizaje fue cementada como un nuevo régimen de verdad. Los medios de comunicación y la publicidad, en los cuales los reportes estadísticos acerca del bajo dominio de lengua de los profesores de inglés en Colombia eran expuestos y promovían la idea de que ellos no hablaban inglés y que por lo tanto el PNB no podía tener buenos resultados. El aprendizaje del inglés se convirtió en una obligación para impactar la subjetividad de los ciudadanos y la crítica a los profesores de inglés colombianos.

Por otro lado, el análisis de documentos oficiales del gobierno y expertos permitieron revisar la construcción de inglés como la lengua del pacto social y oficial para pensar en la educación bilingüe del país. Los temas emergentes arrojan luz sobre las formas en que algunas fuerzas moldearon el establecimiento de un nuevo código de relacionarse al inglés por el beneficio económico y el crecimiento, desarrollo y competitividad del país. Esta 


\section{Afirmación de subjetividades de los profesores de inglés en Colombia \\ a partir de sus discursos de resistencia}

visión es reforzada por expertos tales como los del Consejo Británico, quienes promueven la identidad del hablante nativo para ayudar al profesor de inglés no nativo a ser más efectivo (British Council, 2018) y son quienes sugieren el Marco Común Europeo de Lenguas para estandarizar los niveles de certificación del dominio de inglés en Colombia. En este sentido, se tiene la noción del profesor de inglés como el compañero cooperante del Programa Nacional de Bilingüismo para facilitar su promoción y éxito.

A su vez, en las revistas académicas, los profesores de inglés son los directos interrogadores sobre las maneras como el inglés ha sido promovido. Más importantemente, su posición como sujetos autorizados para hablar de las contradicciones del Plan Nacional de Bilingüismo y sus prácticas excluyentes e injustas son expuestas mientras reclaman para sí condiciones de existencia muy lejos del sistema de ideas impuesto por el PNB y sus promotores. La naturaleza referencial de sus declaraciones da significado a las prácticas contestatarias que correlacionan un dominio de otras prácticas en relación directa o no con las políticas, pero crucial para comprender las formas en que se han percibido a sí mismos y han construido sus subjetividades.

\section{Resultados}

Con la idea de "solo inglés" para promover el bilingüismo en Colombia y la necesidad de profesores de inglés certificados proliferando en los medios de comunicación y en los discursos oficiales y de expertos, actuando como fuerzas que influyen la manera de pensar acerca del bilingüismo en Colombia y de los profesores de inglés que se necesitan para el éxito del PNB, se observó en los documentos cómo una estrategia de dominación funciona para "delinear cómo las cosas y las personas se preparan, se ponen a disposición, y qué medios de ver y decir disponen en los individuos" (Schérer, 2005: 252), para sostener los cuerpos, acostumbrarlos al tenor de una práctica y organizar los mecanismos para servir este instrumento de producción de subjetividad, a través del deseo o la respuesta. La reiteración de ciertos tipos de declaraciones en relación al inglés, tales como "falta de hablantes de inglés", "inglés como una obligación para trabajar y tener éxito", "inglés como un negocio", y "lo nativo como modelo" sirvieron para validar un nuevo tipo de declaraciones para referirse a los profesores de inglés locales como "no bien preparados", "no cualificados" y "quienes no hablan inglés". Esta clase de declaraciones se relacionan con la elaboración de políticas y el razonamiento de expertos para posicionar al inglés como estrategia para la competitividad y profesores locales como facilitadores/técnicos para enfrentar esta demanda. La reducción del bilingüismo a "solo inglés" forzó una visión determinista de profesores que empezaron a ser juzgados por su nivel de inglés sin importar otras dimensiones de su práctica pedagógica. Por otro lado, se pudieron rastrear las prácticas contestatarias a las políticas en las que los profesores locales de inglés cuestionan la base lógica de inglés como lengua de prosperidad y también se oponen a las condiciones idealizadas para la implementación del PNB en las condiciones reales de las escuelas y la enseñanza del inglés en el país. El análisis de los 
archivos develó algunas prácticas de desarrollo profesional en relación a las propias consideraciones de los profesores de inglés acerca de su profesión y la enseñanza del lenguaje que pueden ser representadas como luchas del sí para afirmarse ellos mismos, resistirse y producir conocimiento local relevante.

Luego de una revisión de editoriales, artículos de investigación, artículos de reflexión de profesores universitarios y de profesores de primaria y secundaria de instituciones educativas públicas o privadas, quienes se presentan ellos mismos como investigadores o educadores o profesores de inglés, se identificaron algunas prácticas discursivas y no discursivas como clave de la subjetividad. Estas prácticas se agruparon en apartados bajo los siguientes títulos: convertirse en docentes investigadores, profesores de inglés como educadores, y criticidad como forma de ser.

\subsection{Convertirse en docentes investigadores}

El valor dado a la identidad del docente investigador fue usado para redefinir la formación de profesores en los programas de pregrado y posgrado como un elemento de desarrollo profesional esencial para generar cambios educacionales. El currículo fue enriquecido con materias dedicadas a la investigación de metodologías en pedagogía del lenguaje y lingüística aplicada, junto con técnicas de escritura académica en un momento crucial al final de la década de los 90, en la cual la diseminación de resultados de implementación pedagógica e investigativa empezaron a tomar lugar gracias a la aparición de revistas especializadas. Este tipo de acciones fue patrocinado por diferentes instituciones (universidades, centros de lengua, asociaciones), lideradas por profesores que habían completado un programa de maestría o doctorado en el campo de la enseñanza del inglés, fuera del país, quienes hicieron contribuciones en términos de diseño curricular, pilares educacionales, integrando escenarios para la formación de profesores y la diseminación de resultados de investigación y reflexiones pedagógicas.

\subsection{Profesores de inglés como educadores}

El Plan Nacional de Bilingüismo posicionó a los profesores locales como un equipo para garantizar el éxito del programa. Sin embargo, este equipo no estaba listo debido a su nivel bajo de inglés. Así, los profesores de inglés necesitaban ser evaluados, entrenados, y preparados para seguir las recomendaciones de los expertos. Las reacciones de los profesores de inglés a este tratamiento los hicieron darse cuenta que los lineamientos del PNB los colocaban como técnicos, cuya misión era obedecer el programa sin alterar las condiciones del mismo. Por lo tanto, los profesores confrontaron esta identidad técnica, adoptando la identidad de educadores la cual fue construida por el entendimiento de los profesores sobre su misión, prácticas, experiencias y roles en relación con la educación en un sentido más amplio. Varios temas fueron traídos en las prácticas contestatarias para resistir esta identidad técnica y para indicar cómo el PNB debería ser pensado y aplicado. 
Afirmación de subjetividades de los profesores de inglés en Colombia

a partir de sus discursos de resistencia

\subsection{Criticidad como forma de ser}

Las prácticas contestatarias de los profesores locales de inglés en relación con las nociones del PNB y sus mecanismos para regular la enseñanza del inglés y evaluar a los profesores (guías, estándares, libros de texto, Marco Común Europeo de Referencia para las Lenguas) permitió identificar cómo los profesores de inglés se posicionan ellos mismos desde una perspectiva crítica para oponerse a la identidad nativa idealizada del hablante de inglés, para examinar el valor del inglés declarado y desmantelar la noción de bilingüismo del programa. Al hacerlo, las prácticas discursivas de los profesores favorecieron los dominios sociales, culturales, lingüísticos y políticos para develar la agenda oculta detrás del PNB para la mercantilización y homogeneización del inglés en detrimento de la realidad del país y su riqueza lingüística.

Cuando el PNB fue lanzado, se empezó a promover una idea de sentido común, y esa es la idea de los profesores de inglés locales como partidarios y promotores del programa. Pero es interesante notar un tipo de resistencia en la que la criticidad toma lugar y cuestiona suposiciones que parecen naturales o dadas por hecho. Las formas como los profesores de inglés usaron su conocimiento acerca de la teoría, práctica y condiciones sociales y políticas en la enseñanza de inglés en Colombia para denunciar la segregación sistemática, exclusión y las desigualdades en el programa, implica una línea de pensamiento en la cual ser crítico es una condición de la posibilidad de afirmarse ellos mismos como los más autorizados para hablar sobre la enseñanza de inglés y el bilingüismo en Colombia. Esto envuelve la articulación de diversas prácticas de resistencia para oponerse a los discursos de deber ser dominantes de las políticas, usando las estrategias de poder-conocimiento en las cuales las posibilidades de ser, entender el bilingüismo y legitimar las propias experiencias y saberes de los profesores de inglés están incluidas.

Uno de los aspectos que los profesores de inglés rechazan y cuestionan es la implementación de los estándares y la adopción del Marco Común Europeo de Referencia para las Lenguas sin una perspectiva informada de la realidad colombiana. Para los profesores, fue incomprensible comparar el contexto europeo con el colombiano para copiar este enfoque y usarlo para promover el inglés como la lengua para embarcarse exitosamente en el camino del progreso y la prosperidad. En este escenario, los discursos de los profesores de inglés eran muy ilustrativos para representar las condiciones difíciles de trabajar en las diferentes zonas del país, la falta de inversión y el abandono de algunas escuelas públicas en el país:

Hay un discurso dominante de invertir en inglés y mucha gente lo asocia con prosperidad; una prosperidad que muchos asumen estará disponible para los grandes sectores de la población. Sin embargo, la realidad es que simplemente aprender inglés en el contexto de la educación pública no garantiza a los estudiantes un mejor futuro (Valencia, 2006: 7).

854| Acta Hispanica, Hungría, Supplementum II: 847-858, 2020, ISSN: 1416-7263 


\section{Eliana Garzón Duarte}

Este es el primer indicio de que, para el Ministerio de Educación Nacional, las lenguas indígenas no representan ningún capital, en términos de Bourdieu; [...] el inglés encapsula y representa el ideal de las lenguas extranjeras y es más que suficiente trabajar en él e ignorar otras lenguas (Vélez-Rendón, 2003, citado en Guerrero, 2008: 31).

Un segundo aspecto cuestionado fue la demanda del PNB de un alto nivel de dominio de inglés para todos los ciudadanos sin tener en cuenta las particularidades del contexto, los problemas de orden social y político, y las necesidades y prioridades de muchos sectores en Colombia. Según los profesores de inglés, la estructura jerárquica creada por el Marco Común Europeo, en lugar de generar mejores posibilidades de ser, promueve nuevos mecanismos de medición, marca diferencias y ahonda los vacíos entre los ricos y pobres, los sectores privado y público, y los profesores certificados y no certificados (Ayala y Álvarez, 2005; González, 2009; Usma, 2009).

Un último aspecto problematizado por los profesores de inglés fue la visión deficiente del PNB sobre ellos para enseñar la lengua. Los diagnósticos del PNB fueron basados en los resultados de las evaluaciones que indicaban el bajo dominio de inglés de los profesores y eran usados para descalificarlos y desempoderarlos y también para validar la formación de profesores y los cursos de inglés para mejorar el dominio de la lengua inglesa. Esta consideración reduccionista de lo que un profesor de inglés debe ser, se redujo básicamente a su dominio de lengua, forzó a los profesores no solo a exponer la ausencia del gobierno en patrocinar y apoyar su participación en programas de desarrollo profesional, permiso para asistir a congresos, seminarios o cursos universitarios, sino también a resaltar el compromiso de los profesores de inglés para trabajar por ellos mismos, estudiar, viajar y diseñar cursos (Bastidas y Muñoz, 2011; Bonilla Carvajal y Tejada-Sánchez, 2016; Bonilla y Cruz-Arcila, 2014; entre otros). Algunas prácticas importantes surgieron a partir de esta criticidad en la que resistirse a los discursos de deber ser se convirtió en una lucha de auto-afirmación: se problematizó la noción oficial de bilingüismo, se denunció el silencio de la voz de los profesores en la política pública, los profesores se resistieron a las prácticas dominantes y afirmaron los saberes y las identidades locales.

\section{Discusión y conclusión}

Luego del análisis de los archivos es importante resaltar cómo la construcción de la identidad de los profesores de inglés, como objeto en la ley creados para disciplinar, regular y producir una identidad normalizada, afecta y es afectada por otras fuerzas en las cuales la producción de una respuesta -a través de prácticas de resistencia- hizo visible la capacidad de subjetividad por parte de los profesores. El enfoque de producción de profesores de inglés en el PNB como una práctica política conduce a rastrear formaciones discursivas que este programa realizó como discursos de deber ser para construir una identidad de profesor 


\section{Afirmación de subjetividades de los profesores de inglés en Colombia \\ a partir de sus discursos de resistencia}

de inglés idealizado, una noción de bilingüismo, un ciudadano bilingüe, y también prácticas de enseñanza de inglés que funcionan para encerrar y someter. Primero, los resultados de este estudio a través de la revisión histórica muestran que algunos años antes del lanzamiento del PNB, los profesores locales de inglés no eran atacados por generalizaciones de descrédito y mecanismos de control y disciplina (Foucault, 1977). De hecho, los profesores gozaban de cierto reconocimiento y prestigio. Sin embargo, una vez empezó la implementación del PNB, sus tecnologías de poder-conocimiento alteraron la forma como los profesores de inglés eran percibidos.

Según la discusión de Foucault sobre el carácter doble de sujeción/subjetivación en relación con las prácticas de una mentalidad de autogobierno, el análisis de registros de las prácticas discursivas y no discursivas de los profesores de inglés permitió rastrear algunas prácticas de autoafirmación, trabajo del sí en el que el aparato de subjetividad se experimenta y también, para revisar las prácticas contestatarias en las que la resistencia como una práctica de ejercicio de poder sirvió para problematizar el PNB, cuestionarlo, denunciar sus mecanismos de exclusión, inequidad y normalización de algunas prácticas e identidades. Entre estas prácticas discursivas y no discursivas, convertirse en docentes investigadores, autores, ser críticos y posicionarse como educadores recargó una imagen de bajo estatus con valor positivo que funciona contra la dominación y ofrece formas alternativas de ser y trabajar para visualizar subjetividades más empoderantes para los profesores de inglés.

Lo que esta investigación trajo a superficie son las luchas de los profesores para gobernar su propia existencia y trabajo. El objetivo no es romantizar estas luchas o sugerir que todos los profesores de inglés son oponentes de los discursos dominantes del deber ser. De hecho, los resultados de este estudio se arriesgan a decir que algunos de ellos disfrutan de los mecanismos de control creados por el sistema o se piensan como embajadores del inglés global del norte, replicando y perpetuando estos discursos del deber ser. Lo que se quiere resaltar en esta investigación es que el archivo construido subvierte la sujeción normalizada del PNB y provee un nicho para analizar las complejidades de las subjetividades de los profesores de inglés para adoptar no solo posiciones de identidad, saberes y acciones, sino también sus contradicciones, heridas, miedos y resentimientos. Esta investigación es simplemente un comienzo y una puerta abierta para continuar explorando la subjetividad como un sitio de resistencia.

\section{Referencias bibliográficas}

Atkins, Kim (2005). Self and Subjectivity. Oxford: Blackwell Publishing. https://doi.org/ 10.1002/9780470774847.

Ayala Zárate, Jair - Álvarez V., José Aldemar (2005). A Perspective of the Implications of the Common European Framework Implementation in the Colombian Socio-cultural Context. Colombian Applied Linguistics Journal, 7. 7-26. https:/ / doi.org/10.14483/22487085.162. 
Bastidas, Jesús Alirio - Muñoz Ibarra, Gaby del Socorro (2011). A Diagnosis of English Language Teaching in Public Elementary Schools in Pasto, Colombia. HOW Journal, 18(1). 95-111.

Biehl, Joao - Good, Byron - Kleinman, Arthur (2007). Subjectivity. California: University of California Press.

Bonilla Carvajal, Camilo Andrés - Tejada-Sanchez, Isabel (2016). Unanswered Questions in Colombia's Foreign Language Education Policy. Profile: Issues in Teachers' Professional Development, 18(1). 185-201. https://doi.org/10.15446/profile.v18n1.51996.

Bonilla, Sandra Ximena - Cruz-Arcila, Ferney (2014). Critical Socio-Cultural Elements of the Intercultural Endeavour of English Teaching in Colombian Rural Areas. Profile: Issues in Teachers' Professional Development, 16(2), 117-133. https://doi.org/10.15446/ profile.v16n2.40423.

Castañeda-Peña, Harold - Ubaque, Diego (2017). Teacher Research: Uncovering Professional Identities and Trajectories of Teacher Researchers through Narrative Research - A Colombian Case. International Education Studies, 10(3). 35-45. https://doi.org/10.5539/ies.v10n3p35.

Foucault, Michel (2011). The Courage of the Truth (The Government of Self and Others II). Lectures at The College De France 1983-1984. UK: Palgrave Macmillan.

Foucault, Michel (1988). Technologies of the Self. En: Luther, H. Martin - Gutman, Huck - Hutton, Patrick H. (eds.) 1988. A Seminar with Michel Foucault. Great Britain: Tavistock Publications.

Foucault, Michel (1982). The Subject and Power. Critical Inquiry, 8. 777-795. https://doi.org/10.1086/448181.

Foucault, Michel (1981). Subjectivité et Vérité. Annuaire du Collège de France, 81. Histoire des Systèmes de Pensée. París: Colegio de Francia. 385-389.

Foucault, Michel (1977). Discipline and Punish. The Birth of the Prison. New York: Vintage Books.

Foucault, Michel (1972). The Archaeology of Knowledge and The Discourse on Language. Pantheon Books: New York.

González, Adriana (2009). On alternative and additional certifications in English language teaching: The case of Colombian EFL teachers' professional development. Íkala, revista de lenguaje y cultura, 14(22). 183-209.

Guattari, Pierre Felix (1996). The Guattari Reader. Oxford: Blackwell Publishers Ltd.

Guerrero, Carmen Helena (2008). Bilingual Colombia: What does It Mean to Be Bilingual within the Framework of the National Plan of Bilingualism? Profile: Issues in Teachers' Professional Development, 10(1). 27-45.

Guerrero, Carmen Helena - Meadows, Bryan (2015). Global Professional Identity in Deterritorialized Spaces: A Case Study of a Critical Dialogue Between Expert and Novice 
Afirmación de subjetividades de los profesores de inglés en Colombia a partir de sus discursos de resistencia

Nonnative English Speaker Teachers. Profile: Issues in Teachers' Professional Development, 17(2), 13-27. https://doi.org/10.15446/profile.v17n2.44194.

Guerrero, Carmen Helena - Quintero, Álvaro (2016). Las voces de los maestros frente a las politicas educativas: ¿la ilusión de la democracia? Bogotá: Universidad Distrital Francisco José de Caldas, Centro de Investigaciones y Desarrollo Científico.

Kleinman, Arthur - Fitz-Henry, Erin (2007). The Experiential Basis of Subjectivity: How individuals Change in The Context of Societal Transformation. En: Biehl, Joao - Good, Byron - Kleinman, Arthur (eds.) 2007. Subjectivity. California: University of California Press. 52-65.

Lazzarato, Maurizio (2014). Signs and machines. Capitalism and the Production of Subjectivity. United States: Semiotext.

Macedo, Donaldo - Dendrinos, Bessie - Gounari, Panayota (2003). The Hegemony of English. Boulder, CO: Paradigm.

Pennington, Martha C. (2015). Teacher Identity in TESOL: A Frames Perspective. En: Cheung, Yin Ling - Said, Selim Ben - Park, Kwanghyun (eds.) Advances and Current Trends in Language Teacher Identity Research. New York: Routledge. 16-30.

Schérer, René (2005). A su disposición. En: Zuluaga Garcés, Olga Lucia et al. (eds.). Foucault, la pedagogía y la educación. Pensar de otro modo. Bogotá: Magisterio. 251-318.

Usma Wilches, Jaime (2009). Education and Language Policy in Colombia: Exploring Processes of Inclusion, Exclusion, and Stratification in Times of Global Reform. Profile: Issues in Teachers' Professional Development, 11(1). 123-141.

Valencia Giraldo, Silvia (2006). Literacy practices, texts, and talk around texts: English language teaching developments in colombia. Colombian Applied Linguistics Journal, 8. 7-37. https://doi.org/10.1448 3/22487085.170.

Vélez-Rendón, Gloria (2003). English in Colombia: A sociolinguistic profile. World Englishes, 22(2). 185-198. https://doi.org/10.1111/1467-971X.00287.

Viáfara, John (2016). "I'm Missing Something": (Non) Nativeness in Prospective Teachers as Spanish and English Speakers. Colombian Applied Linguistics Journal, 18(2). 11 24. https://doi.org/10.14483/calj.v18n2.9477. 